\title{
Utilization of Maternal Health Care Among Immigrant Mothers in New York City, 2016-2018
}

\author{
Sheela Maru • Lily Glenn (D) Kizzi Belfon • \\ Lauren Birnie · Diksha Brahmbhatt · \\ Max Hadler · Teresa Janevic · Simone Reynolds
}

Accepted: 21 October 2021 /Published online: 22 November 2021

(C) The Author(s) 2021

\begin{abstract}
Immigrant women represent half of New York City (NYC) births, and some immigrant groups have elevated risk for poor maternal health outcomes. Disparities in health care utilization across the maternity care spectrum may contribute to differential
\end{abstract}

Sheela Maru and Lily Glenn are co-first authors.

Supplementary Information The online version contains supplementary material available at https://doi. org/10.1007/s11524-021-00584-5.

S. Maru

Department of Health System Design and Global Health, Icahn School of Medicine at Mount Sinai, New York, NY, USA

S. Maru · T. Janevic

Department of Obstetrics, Gynecology, and Reproductive Science, Icahn School of Medicine at Mount Sinai,

New York, NY, USA

\section{S. Maru}

New York City Health + Hospitals/Elmhurst, New York, NY, USA

\section{Glenn $(\bowtie) \cdot$ K. Belfon}

Center for Health Equity and Community Wellness, New York City Department of Health and Mental Hygiene, 42-09 28th Street, Long Island City, NY 11101, USA e-mail: lglenn2@health.nyc.gov

\section{Birnie}

Division of Family and Child Health, New York City Department of Health and Mental Hygiene, Long Island City, NY, USA maternal health outcomes. Data on immigrant maternal health utilization are under-explored in the literature. We conducted a cross-sectional analysis of the population-based NYC Pregnancy Risk Assessment Monitoring System survey, using 2016-2018 data linked to birth certificate variables, to explore selfreported utilization of preconception, prenatal, and postpartum health care and potential explanatory pathways. We stratified results by maternal nativity and, for immigrants, by years living in the US; geographic region of origin; and country of origin income grouping. Among immigrant women, 43\% did not visit a health care provider in the year before

D. Brahmbhatt

Weill Cornell Medical College, New York, NY, USA

M. Hadler

Independent Consultant, Brooklyn, NY, USA

T. Janevic

Blavatnik Family Women's Health Research Institute, Icahn School of Medicine at Mount Sinai, New York, NY, USA

T. Janevic

Department of Population Health Science and Policy, Icahn School of Medicine at Mount Sinai, New York, NY, USA

S. Reynolds

Department of Epidemiology and Biostatistics, School of Public Health, SUNY Downstate Health Sciences University, Brooklyn, NY, USA 
pregnancy, compared to $27 \%$ of US-born women (risk difference $[\mathrm{RD}]=0.16,95 \%$ CI $[0.13,0.20]$ ), $64 \%$ had no dental cleaning during pregnancy compared to $49 \%$ of US-born women $(\mathrm{RD}=0.15,95 \% \mathrm{CI}$ $[0.11,0.18])$, and $11 \%$ lost health insurance postpartum compared to $1 \%$ of US-born women $(\mathrm{RD}=0.10$, 95\% CI [0.08, 0.11]). The largest disparities were among recent arrivals to the US and immigrants from countries in Central America, South America, South Asia, and sub-Saharan Africa. Utilization differences were partially explained by insurance type, paternal nativity, maternal education, and race and ethnicity. Disparities may be reduced by collaborating with community-based organizations in immigrant communities on strategies to improve utilization and by expanding health care access and eligibility for public health insurance coverage before and after pregnancy.

\section{Introduction}

The United States (US) is home to the largest population of immigrants in the world, the majority of who live in just 20 major metropolitan areas [1]. The immigrant population in New York City (NYC) is large and diverse, composed primarily of immigrants from Latin America, Asia, and the Caribbean [2]. NYC immigrants speak over 200 languages and account for $36 \%$ of the city's population, or 3 million people [2]. In 2018, half of all births in NYC were to women ${ }^{1}$ born outside the US [3]. Some immigrant women have poorer maternal health outcomes, compared to US-born women, including higher rates of gestational diabetes [4] and severe maternal morbidity $[5,6]$. Disparities in maternal health care utilization may contribute to these outcomes, as suggested by the existing literature, though much of these data are from Europe, and US immigrant maternal health care utilization is underexplored [7].

Access to and utilization of health care across the maternity care spectrum-preconception, prenatal, and postpartum-is important for optimal maternal health. Preconception care is essential for managing

\footnotetext{
${ }_{1}$ Due to limitations in data collection of all pregnancy-capable genders, we consider the terms "mother," "woman," and "maternal" to apply to any person who is pregnant or capable of being pregnant, or who has delivered a child. When citing published research, we use the terms in those publications.
}

chronic conditions that are risk factors for severe maternal morbidity [8]. Timely and adequate prenatal care is the most common recommendation for improving maternal health outcomes [9]. Postpartum care, in the "4th trimester," is important for optimizing long-term maternal health [10]. Compared to US-born women, immigrant women are less likely to have a usual source of care [11], and more likely to have inadequate and delayed initiation of prenatal care $[12,13]$.

Immigrant women may have poorer access to maternal health care due to lack of insurance. Health insurance contributes to health care utilization, and disparities in health insurance status by nativity are well documented [14-17]. Exclusionary health coverage policies, such as Medicaid ineligibility for some immigrant groups, also limit access to care [18]. In NYC, nearly $18 \%$ of immigrants are uninsured compared to $6 \%$ of the US-born population [19].

Additional factors may contribute to lower utilization of health care services among immigrants, including the complex health care system and lack of awareness of available services [20], cultural and language discordance in health care provision [21], and anti-immigrant rhetoric and policies, such as the Trump administration's expanded definition of the public charge rule [22]. Health care utilization among immigrants may also differ based on time in the US, with more time in the US associated with better access to care [23, 24]. Furthermore, research suggests that utilization patterns may vary by country or region of origin, with European immigrants having better rates of utilization than other immigrants [25].

The maternal health service utilization literature contains several gaps. Maternal health service utilization in the preconception period is under-explored, and no literature to date has examined utilization of postpartum services among immigrant populations in NYC. Additionally, most studies have broadly categorized immigrant populations, which likely masks differences within pan-ethnic populations. Understanding immigrant health service utilization patterns by other categorizations, such as region of origin and country of origin income grouping as defined by the World Bank, may elucidate mechanisms of maternal health disparities and highlight immigrant communities with utilization gaps, which may or may not be geographically clustered. Addressing health care underutilization has implications for reducing severe 
maternal morbidity and mortality and improving maternal health across the life course.

In this study, we fill gaps in the literature by examining data from a survey of women who gave birth in NYC from 2016 to 2018, focusing on utilization of preconception, prenatal, and postpartum care by nativity, and for immigrant women, by years in the US, region of origin, and country of origin income grouping. We further seek to explore the factors that have the most impact on these utilization outcomes across the maternity spectrum. Focusing on NYC allows for an examination of utilization patterns across multiple immigrant populations while holding constant the overall socio-cultural, political, and economic context. To guide our analyses, we adapted Yang et al.'s analytical framework for immigrant health service utilization [26]. Our framework (Fig. 1) details the macrostructural/contextual, predisposing, enabling, and health need factors specific to immigrant women in NYC.

\section{Community Engagement}

The study was guided by input from community stakeholders who work with immigrant women in NYC, to better interpret the data and increase utility for community advocacy. Across a series of meetings in each stage of the research process, stakeholders provided feedback on and refined actionable research questions, helped to contextualize results of analyses, and identified avenues for dissemination to policymakers and community members, such as holding community conversations featuring research results tailored to specific immigrant groups.

\section{Methods}

This study emerged from Health Data for New York City (HD4NYC), a partnership between the New York Academy of Medicine and the NYC Department of Health and Mental Hygiene (NYC Health Department). HD4NYC promotes policy-relevant population health research to improve health equity in NYC and emphasizes community engagement throughout the research process.

\section{Data Sources}

We conducted a cross-sectional analysis of data from the 2016-2018 NYC Pregnancy Risk Assessment

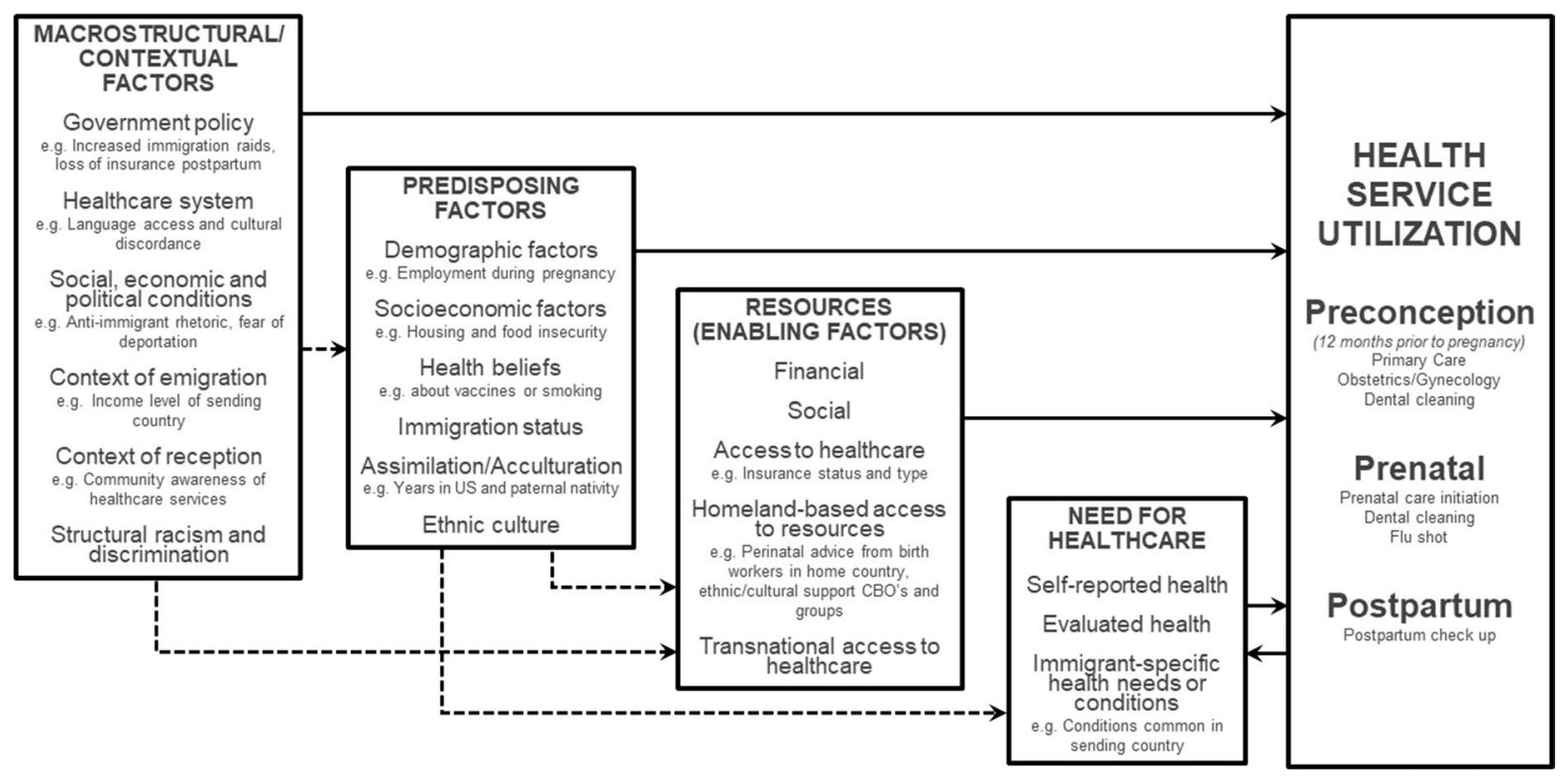

Fig. 1 An analytical framework for maternal health service utilization among immigrant women in NYC. A solid line denotes a direct effect; a broken line indicates that some of the factors within the category have an indirect effect on maternal health service utilization 
Monitoring System (NYC PRAMS), a populationbased surveillance system that collects self-reported data on maternal experiences and behaviors before, during, and shortly after pregnancy, linked with select variables from the birth certificate. The NYC Health Department administered the NYC PRAMS survey in coordination with the Centers for Disease Control and Prevention. The NYC PRAMS survey team drew a stratified random sample without replacement of NYC residents with a live birth from birth certificate data at 2 to 4 months postpartum. Sampled women were sent up to three mail surveys in English, Spanish, or Chinese and, if non-responsive, were followed up with via telephone. NYC PRAMS data were weighted to account for complex survey design, non-response, and non-coverage, to be representative of all NYC residents with a live birth. Non-response weights were calculated using variables related to timing of prenatal care initiation, delivery hospital, and maternal demographic characteristics, including education, age, marital status, race, ethnicity, insurance type, WIC participation, parity, and county of residence. Maternal nativity was not utilized in the calculation of NYC PRAMS survey weights. Annual weighted response rates ranged from 65 to $72 \%$. The NYC Health Department Institutional Review Board reviewed this project and determined it had "exempt" status (IRB no. 19-112).

\section{Measures}

The primary exposure of interest was self-reported maternal nativity (US-born vs. immigrant) on the infant's birth certificate. We categorized immigrant women by years living in the US $(0-4,5-9$, or $10+)$; World Bank geographic region of origin [27] (Europe and Central Asia, Middle East and North Africa, subSaharan Africa, South Asia, East Asia and Pacific, or Latin America and the Caribbean, which we further disaggregated to Caribbean, Central America, or South America using sub-region categories standardized by the United Nations) [28]; and 2018 World Bank income group for country of origin (low-, lower-middle-, upper-middle-, or high-income economies) [27]. The sample size precluded us from categorizing immigrants by individual countries.

We dichotomized maternal health care utilization during the preconception, prenatal, and postpartum periods as reported on the PRAMS questionnaire.
Preconception outcomes were no health care visits in the 12 months before pregnancy overall and by visit type (routine checkup with $\mathrm{OB} / \mathrm{GYN}$, routine checkup with family doctor, primary care visit (OB/ GYN or family doctor), and for dental cleaning). Prenatal outcomes were no or late (third trimester) prenatal care, delayed initiation (second or third trimester) of prenatal care, no flu shot during the 12 months prior to giving birth, and no dental cleaning during pregnancy. Postpartum outcomes were no postpartum check-up and loss of health insurance coverage in the postpartum period.

Covariates were selected to reflect our analytical framework (Fig. 1) as potential explanatory pathways to maternal health care utilization, and by availability in the data sources. Covariates included insurance type during the preconception, prenatal, and postpartum periods (private, Medicaid or other public, other, none, or unknown); race and ethnicity (Latina, White non-Latina, Black non-Latina, Asian or Pacific Islander, or multiracial or other race); maternal education (less than high school, high school/GED, some college/associates degree, or bachelor degree or higher); maternal age $(<20,20-29,30-39$, or $\geq 40)$ and parity (primiparous vs. multiparous); comorbidities 3 months before pregnancy (body mass index (underweight, normal, overweight, or obese), hypertension (yes/no), and diabetes (yes/no)); paternal nativity (US-born, immigrant, or unknown); and experiences of racial bias in the 12 months before giving birth (yes/no). All covariates came from the birth certificate, except for insurance type, pre-pregnancy health status, and experiences of racial bias, which were collected via NYC PRAMS.

We explored missing data for all covariates and found that missing data were more common for prenatal insurance type $(7 \%$ missing) and paternal nativity status (10\% missing). All other covariates had less than $4 \%$ missing data. To determine whether data were missing at random for insurance type and paternal nativity, we created cross-tabulations of the missing and non-missing responses by maternal nativity, years in the US, and World Bank geographic regions. Immigrant respondents were disproportionately represented in the missing insurance data across the maternal health spectrum ( $82 \%$ of the missing data preconception insurance was among immigrants, $79 \%$ for prenatal insurance, and $85 \%$ for postpartum insurance), so we 
added an "unknown" category for insurance type to retain those non-respondents in the regression models. Immigrants tend to be a significant portion of individuals who are uninsured or have transient insurance in the NYC population, so the "unknown" category is a marker for this risk group. Although maternal nativity, years in US, and geographic regions were similarly represented across missing and non-missing responses for paternal nativity, we created an "unknown" category for that covariate because missing birth certificate data on the father can have conceptual meaning regarding paternal involvement. Specifically, evidence has shown that infants with underreported paternal nativity tend to have poorer birth outcomes compared to their counterparts $[29,30]$.

\section{Analysis}

We calculated weighted prevalence estimates and 95\% confidence intervals for all baseline characteristics, covariates, and outcomes of interest. We used chi-square tests to test associations between maternal nativity and baseline demographic, socioeconomic, and macrostructural characteristics. We examined bivariate associations between maternal nativity and health care utilization using predicted marginal effects from logistic regression to calculate crude risk differences (RDs). Unadjusted RDs were calculated for immigrant women overall compared to US-born women, and stratified by years in the US, World Bank geographic regions, and World Bank income groups. Adjusted risk differences were calculated from predicted marginals in the logistic regression model, including covariates individually and combined. We did not conduct a formal causal mediation analysis, as this study is exploratory. Analyses were conducted using SAScallable SUDAAN in SAS Enterprise Guide 7.1 to account for complex survey design, non-response, and non-coverage, enabling the generalizability of findings to all NYC resident births during 2016-2018. We present the results first with an emphasis on the unadjusted risk differences, and then, as an extension of our exploratory approach, the adjusted risk differences with covariates included.

\section{Results}

We compared a variety of demographic, socioeconomic, and macrostructural characteristics by nativity (Table 1). Race and ethnicity differed by nativity, as immigrant women were primarily Latina and Asian or Pacific Islanders. Immigrant women had lower education levels, compared to US-born women, and higher proportions of being unemployed, uninsured, in poverty, participating in WIC, and experiencing food insecurity. Immigrant women were also more likely than US-born women to report experiencing racial discrimination in the year before birth and to have an immigrant father of the infant listed on the birth certificate.

\section{Preconception Outcomes}

Immigrants had lower utilization of preconception health care than US-born women (Fig. 2 for RDs and Supp. Table A1 for prevalence estimates). Notably, immigrant women had higher risk of not having a health care visit during the 12 months before pregnancy $(\mathrm{RD}=0.16,95 \%$ CI $[0.13,0.20])$, not having a primary care visit $(\mathrm{RD}=0.14,95 \%$ CI $[0.10,0.17])$ or regular visit with an OB/GYN $(\mathrm{RD}=0.19,95 \% \mathrm{CI}[0.15,0.22])$, and not receiving a dental cleaning $(\mathrm{RD}=0.18,95 \% \mathrm{CI}[0.15,0.22])$, compared to US-born women. Immigrant women living in the US for the shortest length of time had the highest risk differences, compared to US-born women, with differences in health care utilization decreasing as time spent in the US increased (Fig. 2). Stratified by geographic regions and compared to US-born women, women with the highest risk of no preconception primary care visit were from countries in Central America $(\mathrm{RD}=0.37,95 \%$ CI $[0.31,0.43])$, South Asia $(\mathrm{RD}=0.20,95 \% \mathrm{CI}$ $[0.12,0.28])$, South America $(\mathrm{RD}=0.17,95 \% \mathrm{CI}$ $[0.10,0.24])$, and sub-Saharan Africa $(\mathrm{RD}=0.16$, 95\% CI $[0.06,0.26])$. This was consistent across all preconception health care utilization outcomes. Women from East Asian-Pacific countries were also at higher risk, compared to US-born women, for not having a dental visit $(\mathrm{RD}=0.19,95 \%$ CI $[0.14$, 0.25]) (Fig. 2). The smallest risk differences across most preconception outcomes, as compared to USborn women, were among women from countries 
Table 1 PRAMS sample characteristics by nativity, 2016-2018

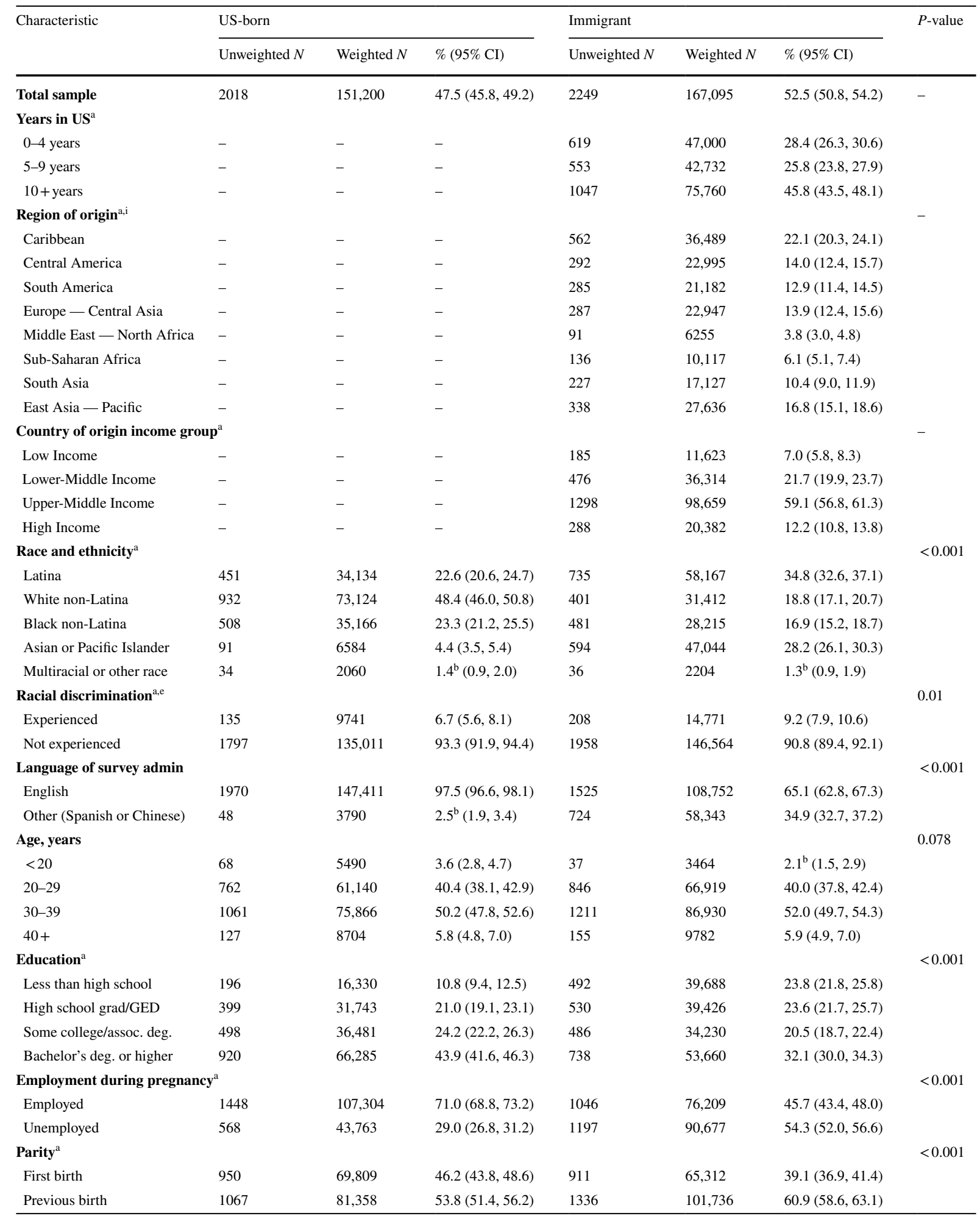


Table 1 (continued)

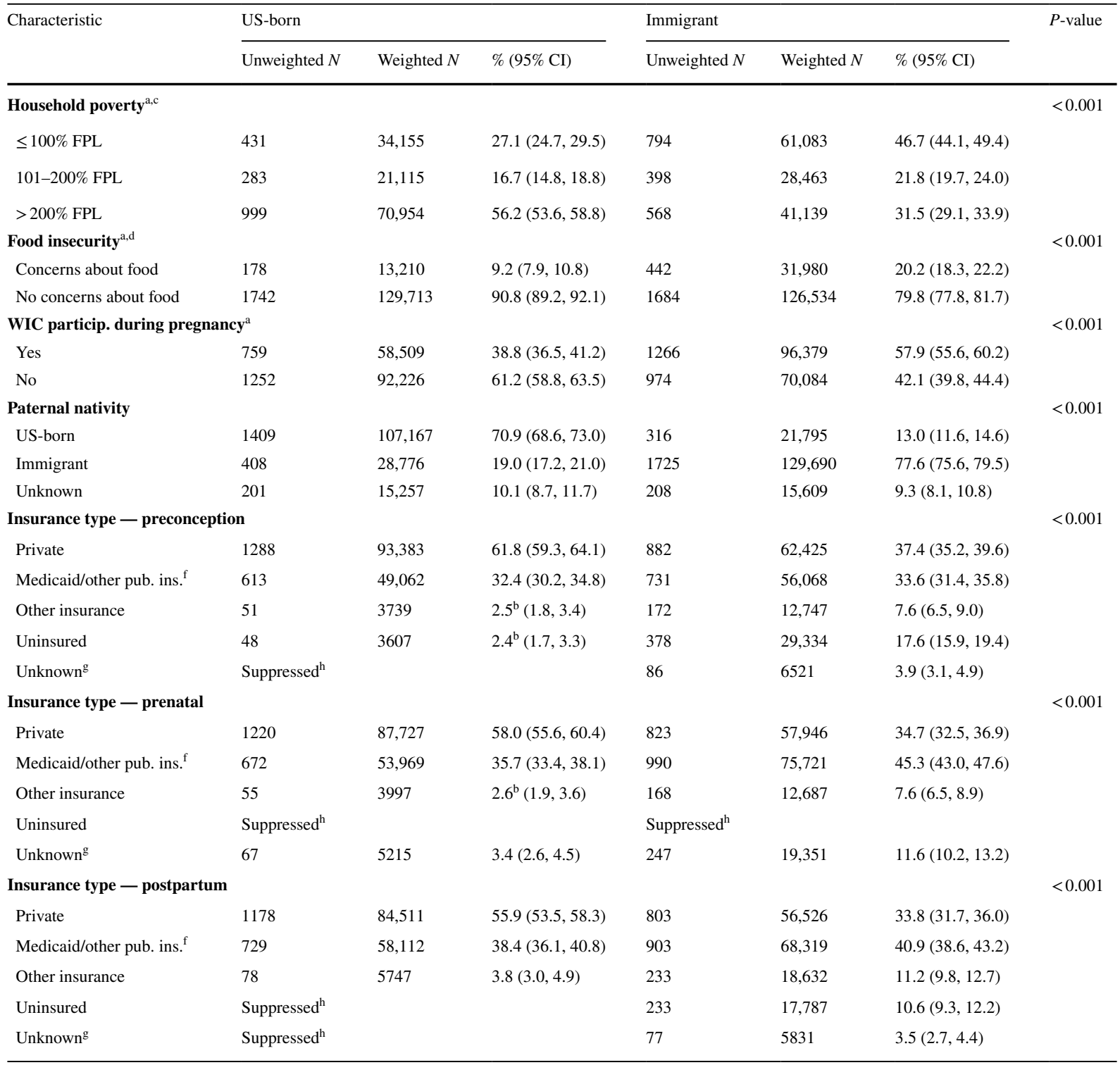

${ }^{a}$ Sample size does not total the sample size overall or by nativity, as some respondents did not answer question

${ }^{b}$ Interpret with caution due to small unweighted sample size $(<60)$

${ }^{c}$ Federal poverty levels (FPL) calculated using Health and Human Services poverty guidelines, 2016-2018

${ }^{\mathrm{d}}$ In the last 30 days

${ }^{\mathrm{e}}$ In the year before birth

${ }^{\mathrm{f}}$ Other public insurance includes Child Health Plus, Family Health Plus, and Family Planning Benefit Program

${ }^{g}$ Unknown insurance includes missing and inconsistent responses

${ }^{\mathrm{h}}$ Results suppressed due to unweighted sample size $<33$

${ }^{\mathrm{i}}$ North American countries are excluded 

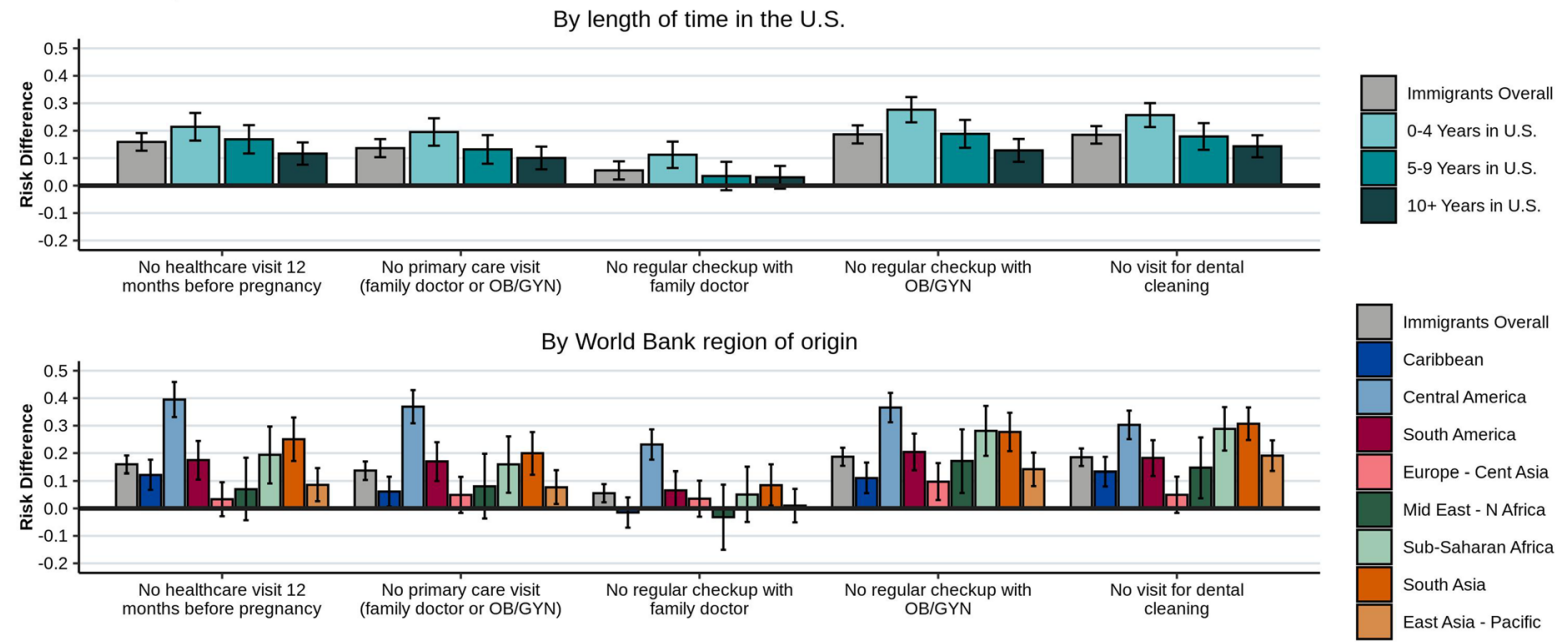

By World Bank income grouping

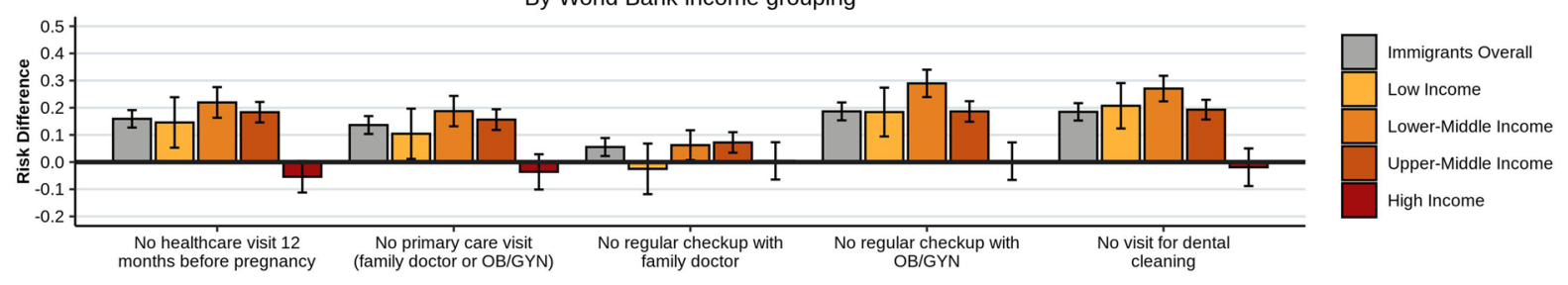

Fig. 2 Preconception health care utilization outcomes for immigrant women giving birth in NYC, 2016-2018. Unadjusted risk differences and $95 \%$ confidence intervals, compared

in Europe and Central Asia. Stratified by income groups and compared to US-born women, immigrant women from lower-middle income countries had the largest utilization differences for most preconception outcomes.

\section{Prenatal Outcomes}

Immigrant women had lower utilization of most prenatal health care outcomes compared to US-born women (Fig. 3 for RDs and Supp. Table A2 for prevalence estimates). Immigrant women were at slightly higher risk than US-born women of not having a prenatal care visit or having a late one (i.e., visit occurred in the third trimester) $(\mathrm{RD}=0.02,95 \% \mathrm{CI}[0.01$, 0.03]) and having a delayed prenatal visit (i.e., first visit occurred after the first trimester) $(\mathrm{RD}=0.02$, 95\% CI $[0.00,0.05])$. Compared to US-born women, immigrant women were at higher risk of not having a dental cleaning during pregnancy $(\mathrm{RD}=0.15$, $95 \%$ CI $[0.11,0.18])$, and the risk differences for no to US-born women, are shown for immigrants overall, and by length of time in the US, World Bank region of origin, and World Bank income grouping

dental cleaning were slightly lower with more time in the US (Fig. 3). We observed no other distinct patterns relating to time in the US for the other prenatal outcomes. Immigrant women were less likely than US-born women to have not received a flu shot during the 12 months before birth $(\mathrm{RD}=-0.05,95 \% \mathrm{CI}$ $[-0.08,-0.01])$, with women in the US for 5-9 years being least likely to have not received a flu shot $(\mathrm{RD}=-0.11,95 \% \mathrm{CI}[-0.16,-0.05])$.

When examined by geographic regions, risk of delayed prenatal care utilization was greatest among women from sub-Saharan African $(\mathrm{RD}=0.15,95 \%$ CI $[0.05,0.25])$, Central American $(\mathrm{RD}=0.14,95 \%$ CI $[0.08,0.20])$, and South American $(\mathrm{RD}=0.09$, 95\% CI $[0.03,0.15])$ countries, compared to USborn women. Women at smallest risk of not having a flu shot during the 12 months before birth, compared to US-born women, were from countries in sub-Saharan Africa $(\mathrm{RD}=-0.19,95 \% \mathrm{CI}$ $[-0.28,-0.09]$ and Central America $(\mathrm{RD}=-0.17$, $95 \%$ CI $[-0.23,-0.11])$, while the women at greatest 


\section{Prenatal Outcomes}
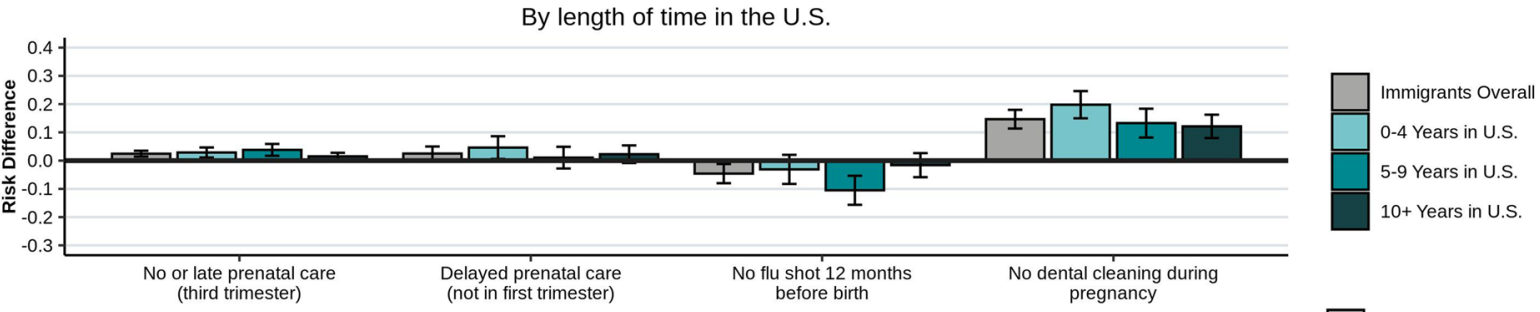

By World Bank region of origin
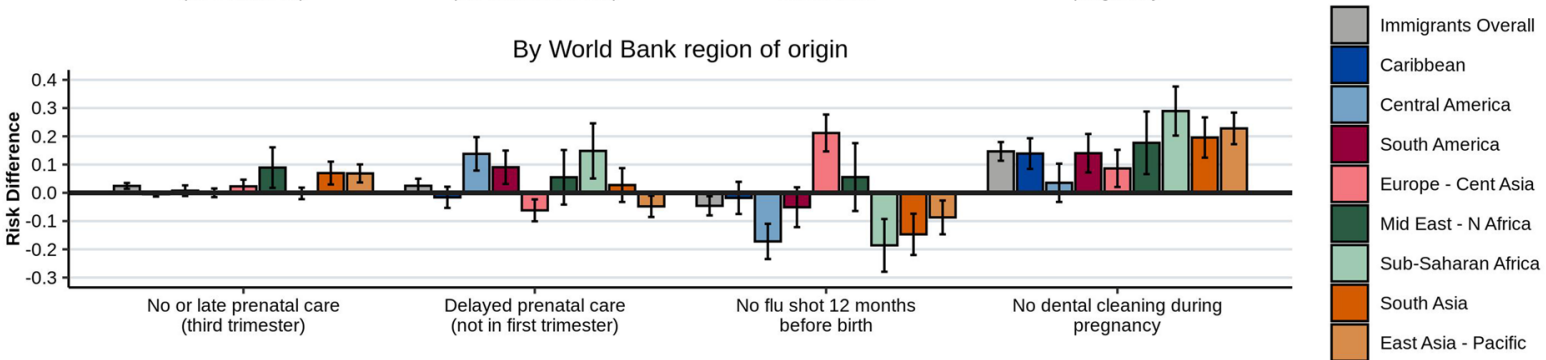

By World Bank income grouping

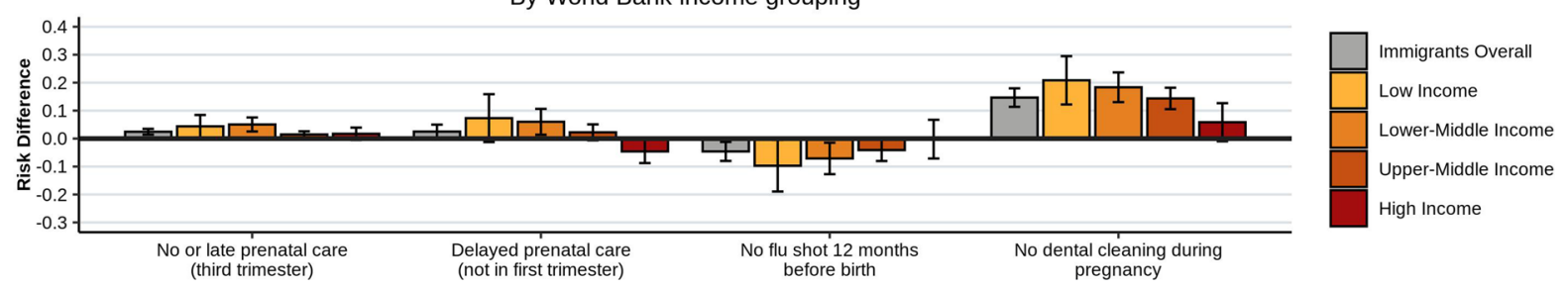

Fig. 3 Prenatal health care utilization outcomes for immigrant women giving birth in NYC, 2016-2018. Unadjusted risk differences and $95 \%$ confidence intervals, compared to US-born

risk of not having a flu shot were from countries in Europe and Central Asia ( $\mathrm{RD}=0.21,95 \%$ CI [0.15, $0.28]$ ). When stratified by income groups, immigrant women from low-income countries had the highest risk of not having a dental cleaning during pregnancy $(\mathrm{RD}=0.21,95 \% \mathrm{CI}[0.12,0.29])$, compared to USborn women, with decreasing risk observed as country incomes increased.

\section{Postpartum Outcomes}

Immigrant women had higher risk of losing insurance during the postpartum period $(\mathrm{RD}=0.10,95 \% \mathrm{CI}$ $[0.08,0.11])$, compared to US-born women. This did not differ based on time in the US (Fig. 4 for RDs and Supp. Table A3 for prevalence estimates). Among all women who lost insurance postpartum (weighted $N=16,596)$, $55 \%$ had no preconception insurance. This was compared to all women who maintained their insurance postpartum (weighted $N=271,976$ ), women, are shown for immigrants overall, and by length of time in the US, World Bank region of origin, and World Bank income grouping

where only $7 \%$ had no preconception insurance. The vast majority (92\%) of women who lost insurance postpartum were immigrants. Postpartum visit attendance was similar between immigrant and US-born women $(\mathrm{RD}=0.01,95 \% \mathrm{CI}[-0.1,0.03])$. However, when categorized by time spent in the US, immigrant women in the US for 0-4 years had somewhat greater risk of not having a postpartum checkup ( $\mathrm{RD}=0.04$, 95\% CI [0.01, 0.08]), compared to US-born women.

When categorized by geographic region, immigrant women from Central American $(\mathrm{RD}=0.35$, 95\% CI $[0.28,0.42])$ and South American countries $(\mathrm{RD}=0.17,95 \%$ CI $[0.12,0.22])$ had the highest risk of losing insurance postpartum, compared to US-born women (Fig. 4). Immigrant women from Central American countries had a slightly higher risk of not having a postpartum appointment $(\mathrm{RD}=0.05,95 \%$ CI $[0.00,0.10])$, compared to US-born women, while immigrant women from East Asian-Pacific countries had lower risk 


\section{Postpartum Outcomes}
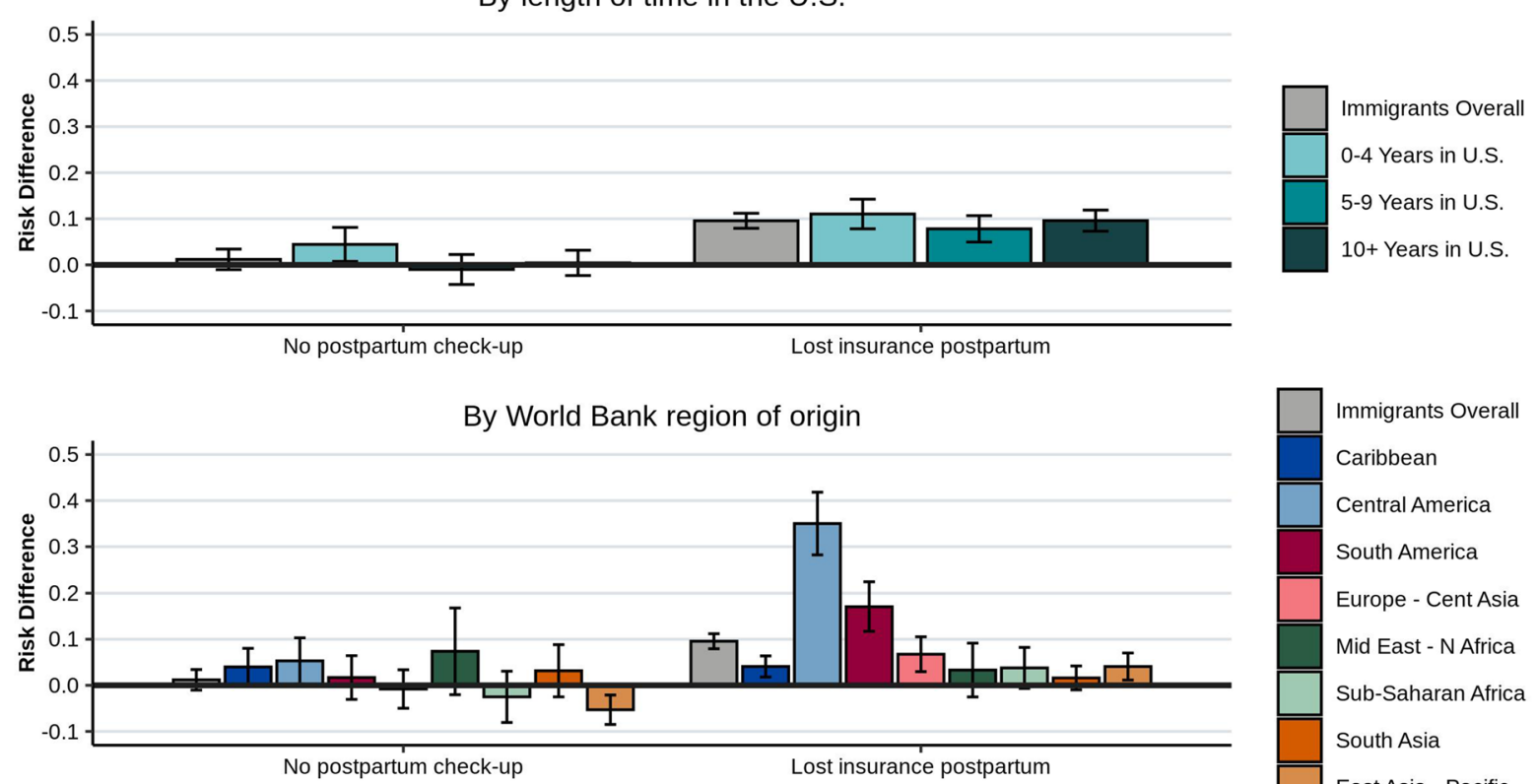

Immigrants Overall

Caribbean

Central America

South America

Europe - Cent Asia

Mid East - N Africa

Sub-Saharan Africa

South Asia

East Asia - Pacific

By World Bank income grouping

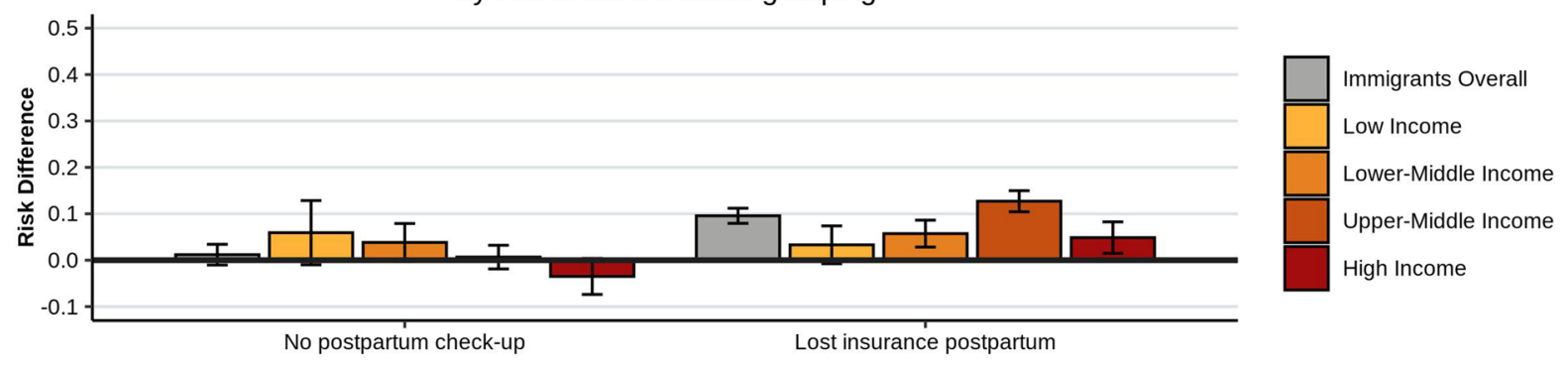

Fig. 4 Postpartum health care utilization outcomes for immigrant women giving birth in NYC, 2016-2018. Unadjusted risk differences and $95 \%$ confidence intervals, compared to US-

$(\mathrm{RD}=-0.05,95 \%$ CI $[-0.08,-0.02])$. Stratified by income group, women from upper-middle income countries had greatest risk of losing insurance postpartum $(\mathrm{RD}=0.13,95 \%$ CI $[0.10,0.15])$, while women from low-income countries had the lowest risk $(\mathrm{RD}=0.03$, 95\% CI $[-0.01,0.07])$, compared to US-born women.

We explored other insurance type trajectories across maternal health periods. From the preconception to prenatal period and from the prenatal to postpartum period, $7 \%$ of respondents overall moved from private insurance to Medicaid or other public insurance and about 3\% moved from Medicaid or other public insurance to private insurance. Differences born women, are shown for immigrants overall, and by length of time in the US, World Bank region of origin, and World Bank income grouping

between immigrant and US-born women were minor or non-existent.

\section{Potential Explanatory Factors}

For most preconception outcomes, adjusting separately for insurance type, paternal nativity, maternal education, and race and ethnicity reduced the RDs between US-born and immigrant mothers, suggesting that these covariates may contribute to health care utilization differences (Supp. Table A4). We observed lowest utilization of preconception health services among mothers with no insurance, those with less than a high school education, those identifying as 
Table 2 Unadjusted and fully adjusted risk differences, immigrants compared to US-born (ref)

\begin{tabular}{|c|c|c|c|c|}
\hline \multirow[t]{2}{*}{ Outcome } & \multicolumn{2}{|c|}{ Unadjusted } & \multicolumn{2}{|c|}{ Adjusted for covariates $^{a}$} \\
\hline & $\mathrm{RD}$ & $95 \% \mathrm{CI}$ & $\mathrm{RD}$ & $95 \% \mathrm{CI}$ \\
\hline \multicolumn{5}{|l|}{ Preconception } \\
\hline No healthcare visit 12 months before pregnancy & 0.16 & $0.13,0.19$ & 0.03 & $0.01,0.08$ \\
\hline No primary care visit (either fam doc or OB/GYN) & 0.14 & $0.10,0.17$ & 0.02 & $-0.02,0.06$ \\
\hline No regular checkup with family doctor & 0.06 & $0.02,0.09$ & -0.00 & $-0.05,0.04$ \\
\hline No regular checkup with OB/GYN & 0.19 & $0.15,0.22$ & 0.06 & $0.02,0.11$ \\
\hline No visit for dental cleaning & 0.18 & $0.15,0.22$ & 0.05 & $0.01,0.09$ \\
\hline \multicolumn{5}{|l|}{ Prenatal } \\
\hline No or late prenatal care (third trimester) & 0.02 & $0.01,0.03$ & 0.01 & $-0.00,0.02$ \\
\hline Delayed prenatal care (not in first trimester) & 0.02 & $0.00,0.05$ & -0.01 & $-0.04,0.03$ \\
\hline No flu shot 12 months before birth & -0.05 & $-0.08,-0.01$ & -0.01 & $-0.06,0.03$ \\
\hline No dental cleaning during pregnancy & 0.15 & $0.11,0.18$ & 0.09 & $0.04,0.13$ \\
\hline \multicolumn{5}{|l|}{ Postpartum } \\
\hline No postpartum check-up & 0.01 & $-0.01,0.03$ & -0.03 & $-0.06,-0.01$ \\
\hline Lost insurance postpartum & 0.10 & $0.08,0.11$ & 0.07 & $0.05,0.09$ \\
\hline
\end{tabular}

a "Lost insurance postpartum" outcome is adjusted for maternal education, race and ethnicity, age, parity, comorbidities three months before pregnancy (diabetes, hypertension, and BMI), experience of racial discrimination, and paternal nativity. All other outcomes are adjusted for the previously listed covariates plus insurance type

Latina, and where the infant's father was an immigrant or paternal nativity was unknown (results not shown). For most prenatal and postpartum outcomes, adjusting for insurance type, paternal nativity, maternal education, and race and ethnicity led to small reductions in the risk differences. Adjustments for age, parity, comorbidities, and experience of racial discrimination had minimal impact on risk differences across preconception, prenatal, and postpartum outcomes.

Table 2 details the adjusted risk differences between US-born and immigrant mothers, including all covariates. Accounting for all covariates in the regression model reduced, but did not eliminate, risk differences for nearly all outcomes.

\section{Discussion}

We found sizeable differences in the utilization of maternal health care between immigrant and US-born women in NYC. These differences were most prominent among recently arrived immigrant women, and those from Central America, South Asia, sub-Saharan Africa, and South America (listed in descending order of RDs, compared to US-born women, for some but not all outcomes). Immigrant women from high-income countries had similar utilization as USborn women. Notably, we reported large differences in preconception care and postpartum insurance loss, points of care previously under-explored in immigrant health literature. Adjusting for insurance type, paternal nativity, maternal education, and race and ethnicity reduced some risk differences comparing immigrants and US-born women, suggesting that some of the potential explanatory mechanisms considered here may warrant further investigation.

Our study builds on previous research highlighting differences in prenatal care utilization between immigrant and US-born women. Late initiation of prenatal care is the most commonly studied utilization measure, and most studies found immigrant women to be at greater risk [31, 32]. We broadened the scope of utilization and showed large differences in preconception care and postpartum insurance loss, together suggesting limited access to non-pregnancy care. Obstetricians and public health leaders increasingly recognize preconception and postpartum periods as crucial to optimizing maternal and infant health outcomes, in addition to long-term maternal health. For example, the preconception period is important for preventing severe maternal morbidity by screening 
for and managing pre-existing conditions [8]. Regular access to care outside of pregnancy also improves access to family planning, allowing for well-informed fertility decisions. Not having health care in the preconception period may lead to unintended pregnancy, which is a strong risk factor for adverse birth outcomes [33]. Finally, non-pregnant periods are an opportunity for improving health literacy and risk awareness; referral to mental health services, smoking cessation, or other substance use services; and promoting a healthy lifestyle [34]. Our findings suggest that immigrant women are less likely to receive this care, compared to US-born women.

A key contribution of our study is our use of country of origin to categorize immigrant women, centering our analysis on shared characteristics such as circumstances of immigration and culture. The standard approach based on the Office of Management and Budget is to categorize race and ethnicity as Hispanic or Latina, non-Hispanic Black, non-Hispanic White, and non-Hispanic Asian, and researchers often examine immigrant vs. US-born outcomes within those groups. Our results underscore how the standard approach can obscure important differences. For example, compared to US-born women, Central Americans had by far the greatest risk of losing insurance postpartum $(\mathrm{RD}=0.35,95 \%$ CI $[0.28,0.42]$ ), and South Asians had greater risk than East Asians of lacking a preconception health visit $(\mathrm{RD}=0.25$, $95 \%$ CI $[0.17,0.33]$ vs. $\mathrm{RD}=0.09,95 \%$ CI [0.03, 0.15]). We also used World Bank income categories to further inform the influence of country-of-origin context. For example, immigrant women from low or lower-middle income countries had the largest differences for most utilization outcomes. These findings provide evidence to advocate for interventions that are tailored to immigrant sub-populations.

We found a suggestion that insurance type was associated with differences in maternal health care utilization. We understand insurance type as a macrostructural factor directly impacting health service utilization as well as a predisposing factor and resource for immigrant women (Fig. 1). In NYC, Medicaid or other public insurance covers nearly half of immigrant women during pregnancy. Undocumented women can enroll in Medicaid when they are pregnant, which gives them insurance access up to 60 days after the end of pregnancy. However, they cannot access Medicaid outside of pregnancy, likely explaining the greater preconception and postpartum utilization disparities. Still, some immigrant groups had higher risk than US-born women of late or no prenatal care. One potential reason is that women who have health insurance in the preconception period may be more likely to have established medical providers, and thus can quickly access care when they discover they are pregnant. For example, researchers found that after Medicaid expansion in Ohio under the Affordable Care Act, pregnant women were more likely to be enrolled in Medicaid prior to pregnancy, and more likely to receive recommended elements of prenatal care [35]. Thus, our finding that immigrant women lack preconception care may influence both health status in pregnancy and the receipt of quality prenatal care, both of which impact health outcomes.

Our data revealed years in the US and paternal nativity as salient to utilization disparities. More recently arrived immigrants had the largest crude disparities, compared to US-born women, in nearly all the utilization outcomes examined. We also found a suggestion that paternal nativity was associated with some utilization outcomes. Both years in the US and paternal nativity are sometimes considered proxies of acculturation. Acculturation describes the process by which individuals adopt the attitudes, values, customs, beliefs, and behaviors of the host country [36]. Time in the US is often studied as a proxy for acculturation, with the assumption that the longer an immigrant lives in the receiving country, the more likely they are to assume its lifestyle and behavioral norms. We consider father's nativity status as a proxy for acculturation due to its potential influence on maternal health behavioral norms. Pregnant immigrants whose partners are US-born fathers are more likely to have health behaviors like US-born mothers, including breastfeeding and smoking. Other mechanisms by which US-born fathers may influence maternal health care utilization include resources and knowledge needed to access the US health system, either through the father himself or his family or social network [37]. However, proxies for acculturation such as years in the US do not capture the "cultural" elements that may be underlying health, nor the macrostructural determinants of immigrant health such as racism, segregation, and exclusionary policies [38]. Years in the US and paternal nativity may reflect macrostructural factors, such as immigration law and Medicaid policy 
as much as acculturation (Fig. 1). For example, time in the US and paternal nativity may also be correlated with an immigration status considered "non-qualified" for federal assistance, including undocumented or unauthorized immigrants and immigrants with temporary visas [39]. Among immigrants with this status, fear of consequences on one's status may discourage accessing care, even care to which they have a right, like pregnancy care in New York.

Adjusting for maternal education partially reduced some utilization disparities. Associations between education and prenatal care utilization are well-established [40]. Education serves as a proxy for socioeconomic status and health knowledge and beliefs [41]. Education also can serve as a proxy for circumstances of migration and reflect early life socioeconomic status in the country of origin. Further, education may reflect current transnational social networks in the country of origin and NYC, which may influence access to knowledge and resources [42]. Culturally tailored interventions should be inclusive of immigrants with less education. Improving access to education in immigrant communities may also represent a point of intervention itself, by increasing socioeconomic opportunity.

Adjusting for race and ethnicity partially attenuated differences in preconception healthcare utilization differences. Race and ethnicity, when interpreted as a social construct, reflects structural and interpersonal racism, which are actionable pathways [43, 44]. Policies to dismantle racism are vital to achieving immigrant health equity. However, race and ethnicity does not entirely account for immigrant utilization differences, suggesting anti-racism policies must be inclusive of immigrants.

\section{Policy Implications}

Our findings of immigrant maternal care utilization disparities inform current policy debates regarding maternal morbidity and mortality, immigration, and Medicaid eligibility. The crisis of high US maternal mortality, particularly among Black women, is in the national spotlight. Federal legislation aims to improve maternal health outcomes and reduce racial and ethnic disparities. The American Rescue Plan Act gives states the option to temporarily extend Medicaid eligibility to 12 months postpartum but does not extend eligibility to immigrants currently ineligible for Medicaid [45]. In New York, this includes unauthorized immigrants, but depending on the state may also include legal permanent residents. Our findings highlight that immigrant women are at risk of missing postpartum care, and undocumented women should be fully included in any extension of postpregnancy coverage. Our findings of disparities in preconception care also inform concurrent discussions around increasing public insurance eligibility that is not conditional on pregnancy. Strategies include removing the 5-year Medicaid waiting period for legal permanent residents, extending Medicaid to all low-income undocumented persons, and universal health coverage. There are approximately 3.5 million undocumented women of reproductive age in the US [46]. Given the sizeable population affected by current exclusions, such policy changes are important to reducing maternal health care utilization disparities.

Support linking immigrants to navigating the health care system, or moreover, simplification of the health care system may reduce disparities, particularly for recent immigrants who have among the lowest utilization rates in our study. These findings support programs for low-income immigrants that provide health navigation, awareness of health access rights, and information on immigration status and health insurance eligibility. The rapid change in immigration policy under the Trump administration and regulations such as the public charge rule have created additional layers of complexity and confusion $[47,48]$. Their effect on immigrant's health care will need to be explored in future studies.

\section{Limitations and Strengths}

A notable limitation to our study is potential participation bias. Lawfully present and less-marginalized immigrant women may be more likely to participate, resulting in attenuation of apparent utilization disparities. Also, the survey was conducted in only three languages, likely resulting in the exclusion of women facing health care access issues due to language, again underestimating disparities. Furthermore, we had little information on mechanisms such as person-centered care, health care discrimination, health knowledge and beliefs, and documentation status. These limitations point to the importance of community-based qualitative research to elucidate barriers to care. Other limitations include cross-sectional study 
design and insufficient data to explore all countries of origin individually. Lastly, we did not conduct a formal causal mediation analysis, so caution is required when interpreting the adjusted analyses.

Despite these limitations, our research has notable strengths. We obtained time in the US and paternal nativity from the NYC birth certificate, unique data often unavailable in studies of immigrant perinatal health. We used novel categories of immigrants, including socioeconomic context of sending country, which are relevant to health disparities research and immigration policy. Finally, we engaged community stakeholders in the development and interpretation of our findings.

\section{Conclusion}

Our study highlights disparities in maternal health care utilization among immigrants giving birth in NYC, with potential explanatory factors of insurance, paternal nativity, maternal education, and race and ethnicity. The most pronounced disparities were during the preconception and postpartum periods, both of which have become increasingly recognized as important in preventing severe maternal morbidity and mortality. Our analysis provides insights into the utilization patterns for specific ethnic sub-populations, which is a strength of data gathered from the diverse population of NYC. We encourage working closely with community-based organizations to identify tailored strategies for improving utilization. Future studies should further explore the reasons for disparities as well as test community-specific interventions to address these disparities. The policy implications of these data include expanding health care access prior to and beyond pregnancy, for which universal health insurance coverage would be a key strategy.

Acknowledgements The authors would like to thank the following stakeholders for their contributions in shaping our analyses and for contextualizing preliminary results: Gabriela Ammann; Amida Castagne; Angela Castillo; Regina Conceicao; Narbada Chhetri; Priscilla Dejesus; Walter Fendrich; Mary Archana Fernandez; Ann Howson; Abigail R. Koch, PhD; Clarisse Kouadjo; Julie Laurence, LMSW; Ray Lopez; Shale Maulana; Brandon Miller; Amitasrigowri S. Murthy, MD, MPH, FACOG; Ilish Neely; Nkiruka Nwokoye; Chanel Porchia-Albert; Rachel Schwartz; Hager Shawkat; Mary-Powel
Thomas; and Alison Whitney. We also thank Elizabeth Kelman and Michele Calvo for their ongoing coordination and support; Mary Huynh, Lisa Bates, and David Siscovick for their guidance; and Hannah Gould, Ana Gallego, Samantha De Leon, Hannah Searing, Yang Jiang, Winfred Wu, and Rishi Sood for reviewing the manuscript. We would like to acknowledge the resilience and strength of immigrant communities and birthing people in NYC.

Funding This study was supported by Health Data for NYC, a partnership of the NYC Department of Health and Mental Hygiene and the New York Academy of Medicine, which is funded by the Robert Wood Johnson Foundation.

Open Access This article is licensed under a Creative Commons Attribution 4.0 International License, which permits use, sharing, adaptation, distribution and reproduction in any medium or format, as long as you give appropriate credit to the original author(s) and the source, provide a link to the Creative Commons licence, and indicate if changes were made. The images or other third party material in this article are included in the article's Creative Commons licence, unless indicated otherwise in a credit line to the material. If material is not included in the article's Creative Commons licence and your intended use is not permitted by statutory regulation or exceeds the permitted use, you will need to obtain permission directly from the copyright holder. To view a copy of this licence, visit http://creativecommons.org/licenses/by/4.0/.

\section{References}

1. Pew Research Center. Key findings about U.S. immigrants. https://www.pewresearch.org/fact-tank/2020/08/ 20/key-findings-about-u-s-immigrants/. Published 2020. Accessed 15 Aug 2021.

2. New York City Mayor's Office of Immigrant Affairs. State of our Immigrant City: MOIA Annual Report for Calendar Year 2020. https://www1.nyc.gov/assets/immigrants/ downloads/pdf/MOIA-Annual-Report-for-2020.pdf. Published 2021. Accessed 22 April 2021.

3. Li W, Onyebeke C, Huynh M, et al. Summary of Vital Statistics 2018. New York, NY: New York City Department of Health and Mental Hygiene, Bureau of Vital Statistics, 2020.

4. Janevic T, Zeitlin J, Egorova N, Balbierz A, Howell EA. The role of obesity in the risk of gestational diabetes among immigrant and U.S.-born women in New York City. Ann Epidemiol. 2018;28(4):242-248. https://doi.org/ 10.1016/j.annepidem.2018.02.006

5. New York City Department of Health and Mental Hygiene, Division of Family and Child Health, Bureau of Maternal, Infant, and Reproductive Health. Severe maternal morbidity rates in New York City, 2013-2014. https:// www1.nyc.gov/assets/doh/downloads/pdf/data/severematernal-morbidity-data.pdf. Published 2018. Accessed 1 Feb 2021.

6. New York City Department of Health and Mental Hygiene. Severe maternal morbidity in New York City, 2008-2012. https://www1.nyc.gov/assets/doh/downloads/ 
pdf/data/maternal-morbidity-report-08-12.pdf. Published 2016. Accessed 1 Feb 2021.

7. Almeida L, Caldas J, Ayres-de-Campos D, Salcedo-Barrientos D, Dias S. Maternal healthcare in migrants: a systematic review. Matern Child Health J. 2013;17(8):134654. https://doi.org/10.1007/s10995-012-1149-x.

8. Howell EA. Reducing disparities in severe maternal morbidity and mortality. Clin Obstet Gynecol. 2018;61(2):387-399. https://doi.org/10.1097/GRF.00000 00000000349 .

9. Osterman MJK, Martin JA. Timing and adequacy of prenatal care in the United States, 2016. Natl Vital Stat Rep. 2018;67(3):1-14.

10 McKinney J, Keyser L, Clinton S, Pagliano C. ACOG Committee Opinion No. 736: optimizing postpartum care. Obstet Gynecol. 2018;132(3):784-5.

11. Seo JY, Kuerban A, Bae SH, Strauss SM. Disparities in health care utilization between Asian immigrant women and non-Hispanic white women in the United States. $J$ Womens Health (Larchmt). 2019;28(10):1368-77. https:// doi.org/10.1089/jwh.2018.7532.

12 Goldfarb SS, Smith W, Epstein AE, Burrows S, Wingate M. Disparities in prenatal care utilization among U.S. versus foreign-born women with chronic conditions. $J$ Immigr Minor Health. 2017;19(6):1263-70. https://doi. org/10.1007/s10903-016-0435-x.

13. Kentoffio K, Berkowitz SA, Atlas SJ, Oo SA, PercacLima S. Use of maternal health services: comparing refugee, immigrant and US-born populations. Matern Child Health J. 2016;20(12):2494-501. https://doi.org/10.1007/ s10995-016-2072-3.

14. Singh GK, Rodriguez-Lainz A, Kogan MD. Immigrant health inequalities in the United States: use of eight major national data systems. Sci World J. 2013;512313. https:// doi.org/10.1155/2013/512313.

15. Tarraf W, Jensen GA, Li Y, Toseef MU, Mahmoudi E, Gonzalez HM. Changes in insurance coverage and healthcare use among immigrants and US-born adults following the Affordable Care Act. J Racial Ethn Health Disparities. 2021;8(2):363-74. https://doi.org/10.1007/ s40615-020-00790-y.

16. Derose KP, Escarce JJ, Lurie N. Immigrants and health care: sources of vulnerability. Health Aff (Millwood). 2007;26(5):1258-68. https://doi.org/10.1377/hlthaff.26.5. 1258.

17 Carrasquillo O, Carrasquillo AI, Shea S. Health insurance coverage of immigrants living in the United States: differences by citizenship status and country of origin. Am J Public Health. 2000;90(6):917-23. https://doi.org/10. 2105/ajph.90.6.917.

18. $\mathrm{Ku} \mathrm{L}$, Matani S. Left out: immigrants' access to health care and insurance. Health Aff (Millwood). 2001;20(1):247-56. https://doi.org/10.1377/hlthaff.20.1. 247.

19. New York City Department of Health and Mental Hygiene. EpiQuery - Community Health Survey 2017. https://nyc.gov/health/epiquery. Accessed 10 April 2021.

20 Hacker K, Anies M, Folb BL, Zallman L. Barriers to health care for undocumented immigrants: a literature review. Risk Manag Healthc Policy. 2015;8:175-83. https://doi.org/10.2147/RMHP.S70173.
21. Escarce JJ, Kapur K. Access to and quality of health care. In: Tienda M, Mitchell F, editors. Hispanics and the Future of America. The National Academies Press; 2006. p. 410-46.

22. Haq C, Hostetter I, Zavala L, Mayorga J. Immigrant health and changes to the public-charge rule: family physicians' response. Ann Fam Med. 2020;18(5):458-60. https://doi. org/10.1370/afm.2572.

23 BeLue R, Miranda PY, Elewonibi BR, Hillemeier MM. The association of generation status and health insurance among U.S. children. Pediatrics. 2014;134(2):307-14. https://doi.org/10.1542/peds.2013-3337.

24 Thamer M, Richard C, Casebeer AW, Ray NF. Health insurance coverage among foreign-born US residents: the impact of race, ethnicity, and length of residence. Am J Public Health. 1997;87(1):96-102. https://doi.org/10. 2105/ajph.87.1.96.

25. Bauldry S, Szaflarski M. Immigrant-based disparities in mental health care utilization. Socius. 2017;3. https://doi. org/10.1177/2378023116685718.

26 Yang PQ, Hwang SH. Explaining immigrant health service utilization: a theoretical framework. SAGE Open. 2016;6(2):1-15. https://doi.org/10.1177/2158244016 648137.

27. World Bank. World Bank country and lending groups. https://datahelpdesk.worldbank.org/knowledgebase/artic les/906519-world-bank-country-and-lending-groups. Published 2020. Accessed 1 Aug 2020.

28. United Nations Statistics Division. Standard country or area codes for statistical use (M49). https://unstats.un. org/unsd/methodology/m49/. Published 2020. Accessed 1 Aug 2020.

29. Ma S. Paternal race/ethnicity and birth outcomes. Am J Public Health. 2008;98:2285-92. https://doi.org/10.2105/ AJPH.2007.117127.

30. Li Y, Luo Z, Holzman C, Liu H, Margerison CE. Paternal race/ethnicity and risk of adverse birth outcomes in the United States, 1989-2013. AIMS public health. 2018;5(3):312-23. https://doi.org/10.3934/publichealth. 2018.3.312.

31. Heaman M, Bayrampour H, Kingston D, et al. Migrant women's utilization of prenatal care: a systematic review. Matern Child Health J. 2013;17(5):816-36. https://doi. org/10.1007/s10995-012-1058-z.

32. Wherry LR, Fabi R, Schickedanz A, Saloner B. State and federal coverage for pregnant immigrants: prenatal care increased, no change detected for infant health. Health Aff (Millwood). 2017;36(4):607-15. https://doi.org/10.1377/ hlthaff.2016.1198.

33. Shah PS, Balkhair T, Ohlsson A, Beyene J, Scott F, Frick C. Intention to become pregnant and low birth weight and preterm birth: a systematic review. Matern Child Health J. 2011;15(2):205-16. https://doi.org/10.1007/ s10995-009-0546-2.

34. ACOG Committee Opinion No. 762: Prepregnancy counseling. Fertil Steril. 2019;111(1):32-42.

35. Adams EK, Dunlop AL, Strahan AE, Joski P, Applegate M, Sierra E. Prepregnancy insurance and timely prenatal care for Medicaid births: before and after the Affordable Care Act in Ohio. J Womens Health (Larchmt). 
2019;28(5):654-64. https://doi.org/10.1089/jwh.2017. 6871.

36. Lopez-Class M, Castro FG, Ramirez AG. Conceptions of acculturation: a review and statement of critical issues. Soc Sci Med. 2011;72(9):1555-62. https://doi.org/10. 1016/j.socscimed.2011.03.011.

37. Cheng ER, Taveras EM, Hawkins SS. Paternal acculturation and maternal health behaviors: influence of father's ethnicity and place of birth. J Women's Health. 2018;27(5). https://doi.org/10.1089/jwh.2017.6439.

38. Viruell-Fuentes EA. Beyond acculturation: immigration, discrimination, and health research among Mexicans in the United States. Soc Sci Med. 2007;65(7):1524-35. https://doi.org/10.1016/j.socscimed.2007.05.010.

39. Perreira KM, Pedroza JM. Policies of exclusion: implications for the health of immigrants and their children. Annu Rev Public Health. 2019;40:147-66. https://doi.org/10. 1146/annurev-publhealth-040218-044115.

40. Blakeney EL, Herting JR, Bekemeier B, Zierler BK. Social determinants of health and disparities in prenatal care utilization during the Great Recession period 2005-2010. BMC Pregnancy Childbirth. 2019;19(1):390. https://doi.org/10.1186/s12884-019-2486-1.

41. Echeverria SE, Pentakota SR, Abraido-Lanza AF, et al. Clashing paradigms: an empirical examination of cultural proxies and socioeconomic condition shaping Latino health. Ann Epidemiol. 2013;23(10):608-13. https://doi. org/10.1016/j.annepidem.2013.07.023.

42. Messias DK. Transnational health resources, practices, and perspectives: brazilian immigrant women's narratives. J Immigr Health. 2002;4(4):183-200. https://doi.org/10. 1023/a:1020154402366.
43. VanderWeele TJ, Robinson WR. On the causal interpretation of race in regressions adjusting for confounding and mediating variables. Epidemiology. 2014;25(4):473-84. https://doi.org/10.1097/ede.0000000000000105.

44. Hardeman RR, Karbeah J. Examining racism in health services research: a disciplinary self-critique. Health Serv Res. 2020;55(Suppl 2):777-80. https://doi.org/10.1111/ 1475-6773.13558.

45. American Rescue Plan Act of 2021, H.R. 1319, $117^{\text {th }}$ Congress (2021-2022).

46. Migration Policy Institute. Profile of the unauthorized population: United States. https://www.migrationpolicy. org/data/unauthorized-immigrant-population/state/US. Published 2021. Accessed 26 Feb 2021.

47. Tolbert J PO, Artiga S. Impact of Shifting Immigration Policy on Medicaid Enrollment and Utilization of Care Among Health Center Patients. https://files.kff.org/attac hment/Issue-Brief-Impact-of-Shifting-Immigration-Policy-on-Medicaid-Enrollment-and-Utilization-of-Careamong-Health-Center-Patients. Published 2019. Accessed 1 Feb 2021.

48. New York State Department of Health, New York City Mayor's Office on Immigrant Affairs, and New York City Department of Health and Mental Hygiene. Fact Sheet: WIC Enrollment Trends in New York City. https://www1. nyc.gov/assets/immigrants/downloads/pdf/fact-sheet-wicenrollment-trends-february-2020.pdf. Published 2020. Accessed 1 Feb 2021.

Publisher's Note Springer Nature remains neutral with regard to jurisdictional claims in published maps and institutional affiliations. 